\title{
High-order balanced M-band multiwavelet packet transform-based remote sensing image denoising
}

\author{
Haijiang Wang ${ }^{1,2}$, Jingpu Wang ${ }^{3 *}$, Hai Jin², Lihong Li ${ }^{1}$, Dongli Jia' ${ }^{1}$, Yongqiang Ma ${ }^{1}$, Yongmei Liu ${ }^{4}$, Fuqi Yao ${ }^{5}$ \\ and Qinke Yang ${ }^{4}$
}

\begin{abstract}
This article proposes high-order balanced multi-band multiwavelet packet transforms for denoising remote sensing images. First, properties of several wavelet transforms and their relationships are analyzed. The article then presents theoretical principles and a fast algorithm for constructing high-order balanced multi-band multiwavelet packet transforms. The remote sensing image denoising method based on this transform scheme is then described, and its utility is demonstrated by illustrative results of its application to denoise remote sensing images. The method provides clear improvements in denoising quality, due to the balanced order or band number, consistently outperforming traditional wavelet transform-based methods in terms of both visual quality and evaluation indicators. The method also incurs reasonable computational costs compared with the traditional methods.
\end{abstract}

Keywords: Remote sensing image, Denoising, Multiwavelet packet transform, Multi-band, Balanced

\section{Introduction}

Remote sensing imaging has become a powerful technique for exploring and obtaining knowledge of numerous phenomena. However, during acquisition and transmission processes, the images are often contaminated by noise, which impairs their visual quality and limits the precision of subsequent processing steps, such as classification, target detection, and environmental monitoring. Thus, remote sensing image denoising applications have attracted growing interest. Transform domain denoising methods have shown remarkable success in the last decade. An assumption typically underlying these methods is that signal can be sparsely represented in the transform domain. Hence, by preserving the few high-magnitude transform coefficients that convey most of the true signal energy and discarding the rest, which are mainly due to noise, the true signal can be effectively estimated. The sparsity of the representation depends on both the transform used and the true signal's properties $[1,2]$.

\footnotetext{
* Correspondence: wangjp@ldu.edu.cn

${ }^{3}$ School of Resources and Environmental Engineering, Ludong University, Yantai 264025, People's Republic of China

Full list of author information is available at the end of the article
}

The wavelet transform (i.e., 2-band scalar wavelet transform) can provide good sparsity for spatially localized details, and a number of advanced denoising methods based on them have been developed [2-4]. For example, the wavelet thresholding approach popularized by Donoho is now widely used in scientific and engineering applications [2]. The best image denoising systems include filters with symmetric and compact-support properties, which can effectively extract features and eliminate artifacts. Unfortunately, however, no nontrivial, symmetric, compact-support, orthogonal scalar wavelet transforms are available $[1,5]$.

Multiwavelet transforms (i.e., 2-band multiwavelet transforms or 2-band MWTs) have several advantages over scalar wavelet transforms, because they can simultaneously possess all the above properties $[5,6]$. Since these properties are highly significant in image processing, multiwavelets have attracted considerable research interest and shown superior denoising performance over scalar wavelets in various studies [6-10] (see the diagram about the development of wavelet transforms in Fig. 1). Sveinsson et al. [11] and Wang et al. [12] applied 2-band MWT in remote sensing image denoising and found that they generally outperform 2-band scalar wavelet transforms
Springer

(c) 2016 Wang et al. Open Access This article is distributed under the terms of the Creative Commons Attribution 4.0 International License (http://creativecommons.org/licenses/by/4.0/), which permits unrestricted use, distribution, and reproduction in any medium, provided you give appropriate credit to the original author(s) and the source, provide a link to the Creative Commons license, and indicate if changes were made. 


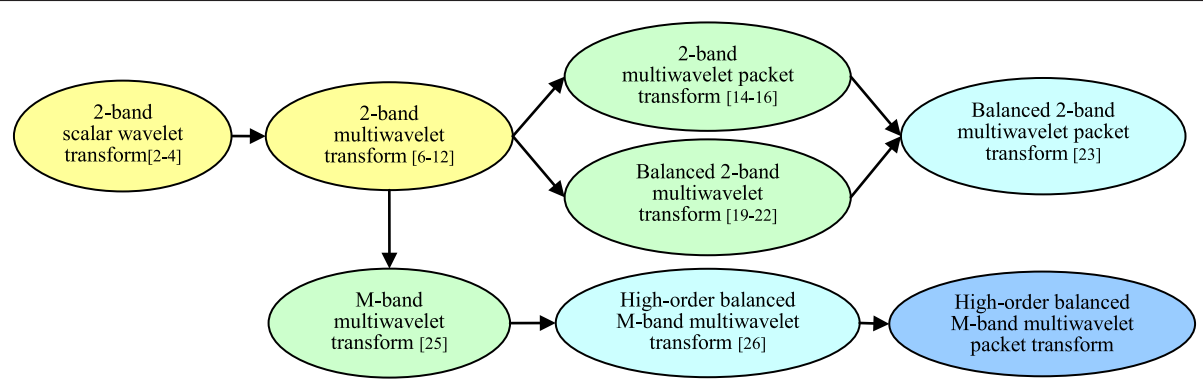

Fig. 1 Development of wavelet transforms and relationships among different kinds of wavelet transform schemes. The transform schemes marked with light green color are compared with our proposed scheme (with light blue color) in our evaluation experiment of denoising performance

in both visual quality and objective evaluation. Further improvements of 2-band MWT could potentially be obtained by taking into account the properties of the signal to be analyzed, but all the cited transforms use the unchangeable transform pattern for diverse images. That is, as illustrated in Fig. 2a, the decomposition at each decomposition level is only applied to the low-frequency component of signals and does nothing to the other, relatively high-frequency components, although this partitioning is not suitable for all signals with different behaviors across the frequency domain $[1,13]$.

Multiwavelet packet transforms (i.e., 2-band multiwavelet packet transforms or 2-band MWPT), as one extension of 2-band MWT, provide an effective mean to select a suitable decomposition pattern corresponding to an analyzed signal. As shown in Fig. 2b, a 2-band MWPT offers a finer frequency domain partition than a 2-band MWT, especially in the high-frequency domain. Hence, numerous subsets corresponding to different multiwavelet bases (or packets) can be found in its partition point set, one of which will match the properties of the analyzed signals better than all the others. The 2-band MWPT based on this subset (the best packet) can provide a better sparse representation of the signal than those based on other subsets (including the 2-band MWT, as the subset it uses is just one of these subsets) [1,13]. Martin et al. [14] and Wang [15] introduced 2-band MWPTs to image compression and texture segmentation, respectively, and showed that they exhibit performance generally superior to 2-band MWT. Liu et al. proposed a choice algorithm of the best multiwavelet packet and found that the 2-MWPT based on the algorithm can generally obtain better denoising result than 2-band MWT at the same condition [16]. Developing a 2-band MWT scheme into its "packet" version is an effective way to improve performance (as shown in Fig. 1), however, the problem of balanced or high-order balanced must be addressed when using them in practical applications.

The balanced order $\rho$ of a multiwavelet system corresponds to its ability to represent images sparsely $[17,18]$. Recent studies show that the 2-band MWTs based on balanced $(\rho=1)$ multiwavelets (i.e., balanced 2-band MWT, as shown in Fig. 1) consistently outperform those based on unbalanced $(\rho=0)$ counterparts (i.e., 2-band MWT) in image denoising [19-22]. Also, balanced 2-band MWPT schemes derived by developing the balanced 2-band MWT into "packet" versions also consistently outperform (unbalanced) 2-band MWPT in seismic data compression and denoising [23]. However, there have been no published indepth studies on the relationship between the balanced order $\rho$ and sparse representation ability of balanced $(\rho=1)$ or high-order balanced $(\rho>1)$ 2-band MWPT (or even MWT). More importantly, although the 2-band MWPTs overcome many shortcomings of wavelet transforms, they

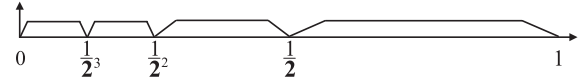

(a)

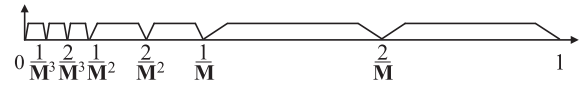

(c)

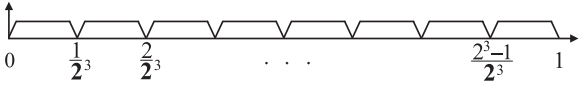

(b)

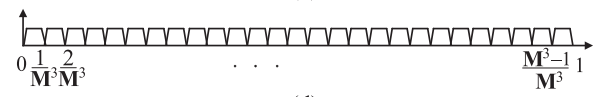

(d)

Fig. 2 The ideal frequency domain portioning patterns offered by different multiwavelet transforms with normalized highest frequency, taking $M=3$ and $k=3$ ( $k$ refers to a decomposition level) as an example. a For a 2-band multiwavelet transform, the ideal partition points are only in the set $\left\{1 / 2,1 / 2^{2}, \cdots, 1 / 2^{k}\right\}$. b For a 2 -band multiwavelet packet transform, they are in the set $\left\{1 / 2^{k}, 2 / 2^{k}, \cdots, 2^{k}-1 / 2^{k}\right\}$. c For an M-band multiwavelet transform, they are in the set $U_{i=1}^{k}\left\{1 / M^{i}, 2 / M^{i}, \cdots, M-1 / M^{i}\right\}$. d For an $M$-band multiwavelet packet transform, they are extended to the set $\left\{1 / M^{k}, 2 / M^{k}, \cdots\right.$, $\left.M^{k}-1 / M^{k}\right\}$ 


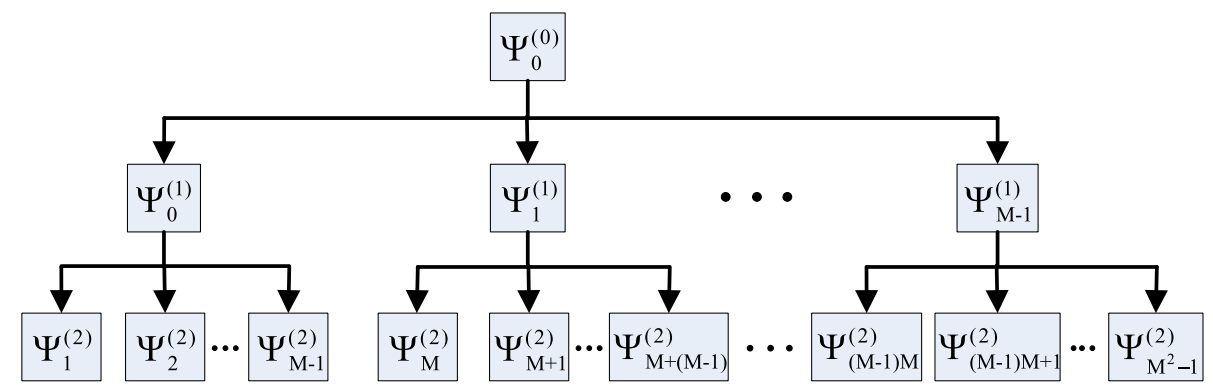

Fig. 3 Library of M-band multiwavelet packet basis functions, in 3-level decomposition

retain the "2-band" weakness of the latter, especially in spatial-frequency tiling, which has triggered great interest in their extension [1].

As another extension of 2-band MWT, M-band $(\mathrm{M} \in$ $\mathbb{Z}$ and $M>2$ ) MWTs provide greater flexibility in spatialfrequency tiling and more robust sparse representation $[1$, 24] (see Fig. 1). As shown in Fig. 2c, the ideal partition point set of an M-band MWT is denser than that of a 2-band counterpart (see Fig. 2a). M-band MWT has been shown to outperform 2-band MWT in terms of both visual quality and objective indicators in image fusion [25]. Similarly, highorder balanced M-band MWTs reportedly outperform (unbalanced) M-band MWT in image compression [26]. Potentially, a high-order balanced M-band MWT may also be further improved (like a 2-band counterpart) by using a "packet" version (i.e., an M-band MWPT, as shown in Fig. 1). As shown by the theoretical partition pattern of an M-band MWPT in Fig. 2d, it may provide a finer partitioning pattern and better matching subset (or more effective representation) for an analyzed signal, relative to a 2-band counterpart. M-band MWPTs may offer great potential for image processing, but substantial extension of both their fundamental theory and convenient methodology are required. Thus, here we present basic principles of, and fast algorithms for, highorder balanced M-band MWPT by developing the highorder balanced M-band MWT presented in [26], according to key theory of developing balanced 2-band MWT into "packet" versions presented in [23]. We also evaluate their performance in remote sensing image denoising in comparison with the 2-band MWPT presented in [14-16], the balanced 2-band MWPT in [23], the M- band MWT in [25], and the high-order balanced M-band MWT in [26] (see Fig. 1). Moreover, we systematically analyze the impact of the balanced order $\rho$ and band number $M$ on the sparse representation ability of MWPT.

The rest of the paper is organized as follows. In Section 2, we analyze the basic principles and fast algorithm of highorder balanced M-band MWPT based on the relative theory of M-band MWT. In Section 3, we present the denoising method based on the proposed transform, and evaluate the denoising performance of the method by using both synthetic and real noisy remote sensing images in comparison with the method based on the transforms recently proposed. We also analyze the impacts of the balanced order $\rho$ and band number $\mathrm{M}$ on their denoising performance. We summarize and discuss the work in Section 4.

\section{High-order balanced M-band multiwavelet packet transforms}

Classical wavelet transforms cannot provide many key properties simultaneously, as they establish a multiresolution analysis frame using a single scale function $[1,5,6]$. In contrast, multiwavelet transforms use $r(r \in \mathbb{Z}$ and $r>1)$ scale functions ( $r$-multiplicity) for this work, thus reducing the number of constraint conditions and increasing the freedom in design. The $r$ scale functions are denoted in vector form, i.e., $\Phi_{0}(t)=\left[\phi_{0,0}, \phi_{1,0}, \cdots\right.$, $\left.\phi_{r-1,0}\right]$. An M-band $r$-multiplicity multiwavelet system has M-1 wavelet function vectors denoted as $\Phi_{i}(t)=\left[\phi_{0, i}\right.$, $\left.\phi_{1, i}, \cdots, \phi_{r-1, i}\right](1 \leq i<\mathrm{M})$. If (and only if) such a system is orthogonal, the $\Phi_{i}(t)$ meets the following condition [24, 26],

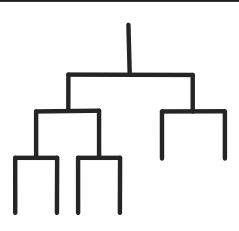

(a)

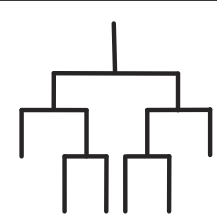

(b)

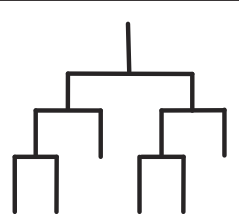

(c)

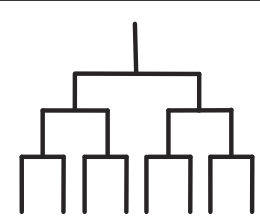

(d)

Fig. 4 Decomposition patterns of different M-band multiwavelet packet transforms (taking $M=2$ and $k=3$ as an example). The pattern in (a) corresponds to the standard M-band multiwavelet decomposition scheme and that in (d) corresponds to a kind of multiwavelet decomposition scheme that presents the finest frequency-domain partition. The patterns in (b-c) correspond to two other possible decomposition schemes respectively 


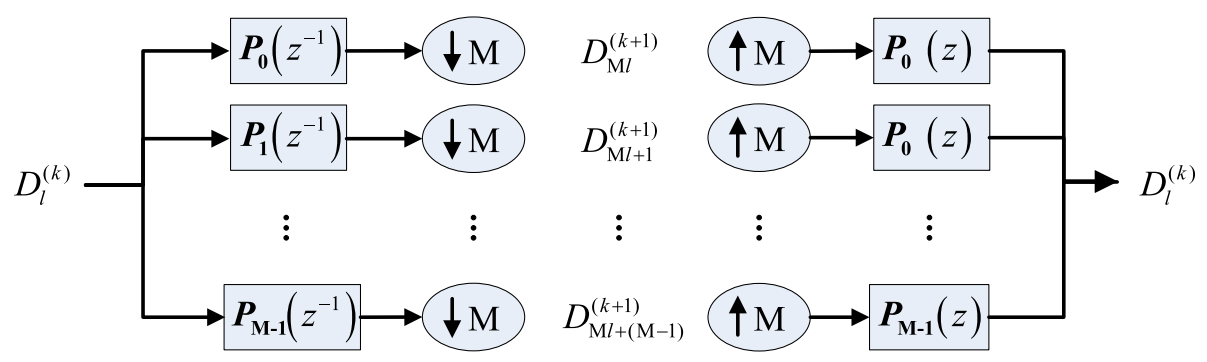

Fig. 5 The decomposition and reconstruction of the M-band multiwavelet packet transform

$$
\Phi_{i}(x)=\sqrt{\mathrm{M}} \sum_{n \in \mathbb{Z}} P_{i}(n) \Phi_{0}(\mathrm{M} x-n)
$$

Here, $P_{i}(n)$ is a vector filter bank of $r \times r$ and $n \in \mathbb{Z}$ (the same as below). We can get the following M-band multiwavelet transform algorithm after developing the corresponding algorithm of the classical wavelet transform (i.e., Mallat algorithm) [24, 26],

$$
\begin{aligned}
& C_{i}^{(k+1)}(n)=\sqrt{M} \sum_{m \in Z} P_{i}(m) C_{0}^{(k)}(M n+m) \\
& C_{0}^{(k)}(m)=\sqrt{M} \sum_{i \in Z} \sum_{n \in Z} P_{i}^{T}(n) C_{i}^{(k+1)}(M n+m) .
\end{aligned}
$$

Here, $m, n \in \mathbb{Z}$ and both are shift parameters, while $C_{i}^{k}$ is an $r$-dimensional vector (the same as below).

A multiwavelet system is said to be balanced of order $\rho$ if its lowpass and highpass filters preserve and cancel, respectively, all the monomial polynomial signals of order less than $\rho$. The order of a multiwavelet system corresponds to its ability to effectively represent the information of the macroscale change trend and local textures of a signal $[17,18]$. If $P_{i}(n)$ meets the constraint condition corresponding to each property above (e.g., $\rho$-order balanced, orthogonality), the balanced or highorder balanced M-band MWPT could be constructed by developing the above $\mathrm{M}$-band multiwavelet transform algorithm. Their basic principles and fast algorithms are present as follows.
2.1 Basic principles

Let $\Psi_{i}(t)=\Phi_{i}(t)(0 \leq i<\mathrm{M})$, and define

$$
\Psi_{\mathrm{M} l+i}=\sqrt{\mathrm{M}} \sum_{n \in \mathbb{Z}} P_{i}(n) \Psi_{l}(\mathrm{M} x-n)
$$

M-fold rescaling and translation of these functions yield a function library $X=\left\{\Psi_{l}^{(k)}=\mathrm{M}^{-k / 2} \Psi_{l}\left(\mathrm{M}^{-k} x-n\right)\right\} \quad(0 \leq l<$ $\left.\mathrm{M}^{k}\right)$. As shown in Fig. 3, the library can be viewed in terms of an M-fold complete tree, and the $l$ th function at the $k$ th decomposition level $\Psi_{l}^{(k)}$ yields $\mathrm{M}$ functions at the $k+1$ level, i.e., $\Psi_{\mathrm{M} l+i}^{(k+1)}(0 \leq i<\mathrm{M})$.

The library is overcomplete, and many complete orthogonal basis sets can be found by properly selecting different subsets in the library with an appropriate parameter set $\{k$, l3.. A complete orthogonal basis set corresponds to a kind of multiwavelet packet transform scheme, a subset of the library and a parameter set $\{k, l\}$.. Each subset can be viewed in terms of an $\mathrm{M}$-fold tree structure. Examples of possible basis sets are shown in the trees in Fig. 4. The tree in Fig. 4a selects the subset of the library with the parameter set $\{1 \leq l<\mathrm{M}, 0 \leq k<3\} \cup\{0 \leq l<\mathrm{M}, k=3\}$ and corresponds to the standard M-band multiwavelet decomposition scheme $[5,6,24]$. The tree in Fig. $4 d$ selects the subset with the parameter set $\left\{0 \leq l<\mathrm{M}^{k-1}, k=3\right\}$ and corresponds to a kind of multiwavelet decomposition scheme that presents the finest frequency domain partition.

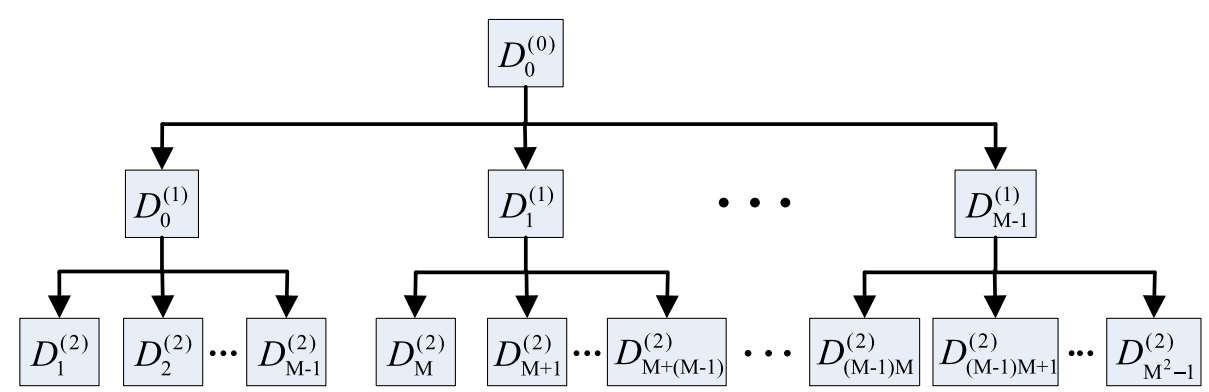

Fig. 6 Relationship of the transform domain coefficient at different decomposition levels in the M-band multiwavelet packet transform, taking $k=3$ as an example 


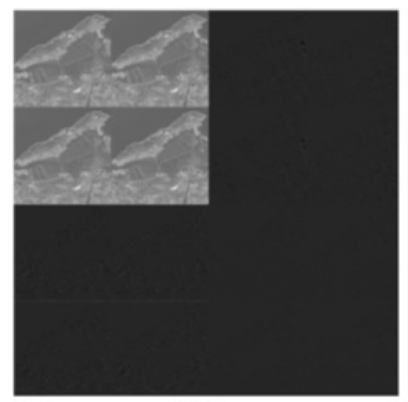

(a)

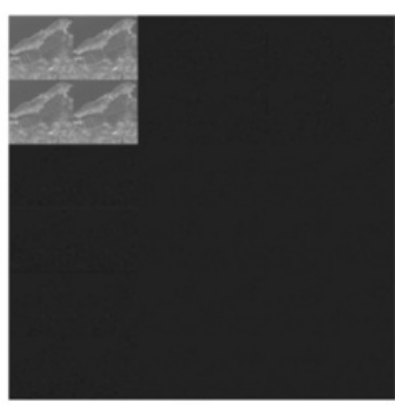

(b)

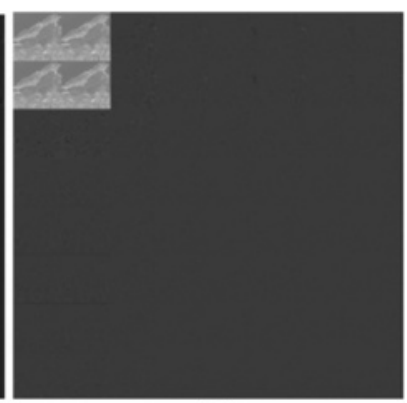

(c)

Fig. 7 Illustrative results of (1-level) decomposition by the M-band multiwavelet packet transform of a remote sensing image, with $M=2$ (a), 3 (b), and 4 (c)

For any analyzed dataset, there is a basis set (the best basis set) that can best represent the spatial and frequency domain information in the dataset, and the corresponding "best" multiwavelet packet transform provides the most effective representation of the dataset (denoted as MWPT at below). The best basis set is found by using a cost function searching algorithm that seeks the best subset for some application in a set with tree structure [1,13]. For example, a searching algorithm that uses information entropy as a cost function can find the most informative basis subset and consistently perform effectively in image denoising $[1,13]$. Since the overcomplete library of the basis sets generated by the high-order balanced M-band multiwavelet packet transform can also be viewed in terms of a tree structure, we used entropic cost function searching algorithms to find both the best basis set corresponding to test datasets and for establishing the searching algorithm.

\subsection{Fast algorithm}

Defining $D_{i}^{(0)}=C_{i}^{(0)}(0 \leq i<\mathrm{M})$, a fast algorithm for decomposition of the M-band multiwavelet packet transform is shown in Eq. (5). Reconstructions are the inverse process of decompositions, and its fast algorithm is shown in Eq. (6). Based on the expression, its clear procedures in $\mathrm{Z}$ transform domain are illustrated in Fig. 5, where $\boldsymbol{P}_{i}(z)$ is the $\mathrm{Z}$ transformation of $P_{i}(n)$.

$$
\begin{aligned}
& D_{M l+i}^{(k+1)}(n)=\sqrt{M} \sum_{m \in Z} P_{i}(m) D_{l}^{(k)}(M n+m) \\
& D_{l}^{(k)}(m)=\sqrt{M} \sum_{i=0}^{M-1} \sum_{n \in Z} P_{i}(n) D_{M l+i}^{(k+1)}(M n+m)
\end{aligned}
$$

Using the decomposition algorithm above, one can obtain a complete $\mathrm{M}$-fold tree including information on all

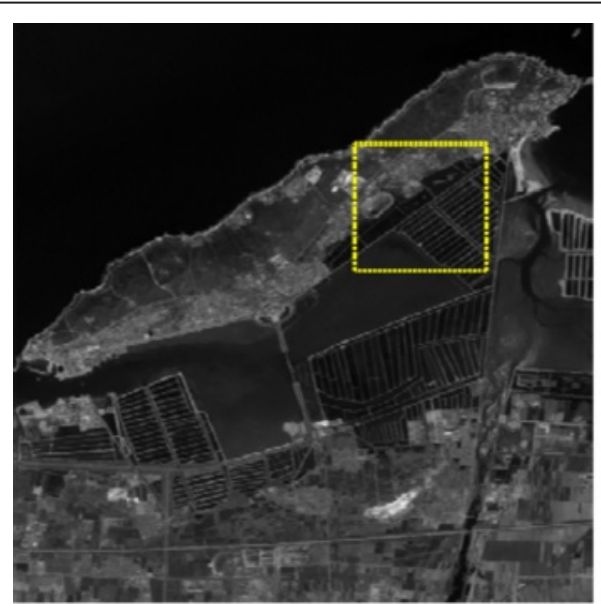

(a) Original noiseless SPOT-5 image.

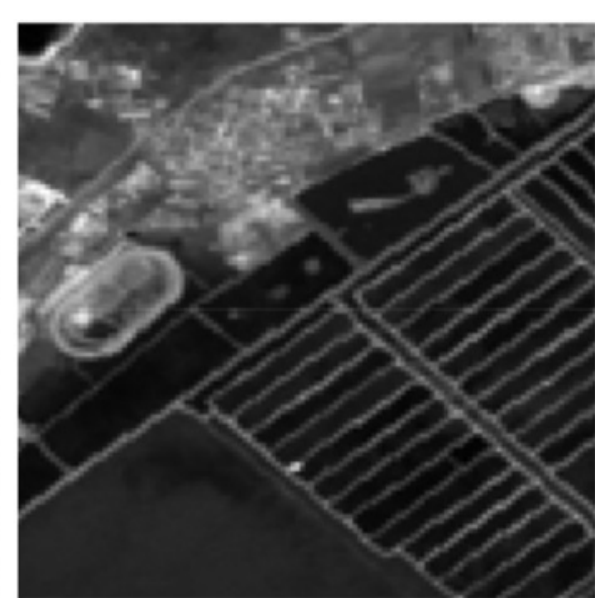

(b) A close-up of (a).

Fig. $\mathbf{8}$ a Original noiseless remote sensing test image and $\mathbf{b}$ a close-up of (a) regarding the region surrounded by the rectangle in (a). The noisy images and denoising results relevant to the part are shown in Figs. 9, 10, 11, and 12 for clear comparison 
its nodes $D_{l}^{(k)}\left(1 \leq l<\mathrm{M}^{k}\right)$, as shown in Fig. 6. $D_{l}^{(k)}$ are multiwavelet domain coefficients containing spatial information of the included frequency bands (see Fig. 2d). After decompositions, we can use the cost function-searching algorithm to find the best basis set $\left\{D_{l}^{(k)}\right\}$ among all the possible basis sets, which can represent the spatial and frequency domain information of the analyzed dataset more effectively than all other basis sets. Use of this best basis set-based multiwavelet packet transform in image processing applications should provide optimal quality results.

Information on the root node $D_{0}^{(0)}$ in the tree illustrated in Fig. 6 can be obtained by $r$-dimensional vectorization of the analyzed datasets. Using the balanced or high-order balanced M-band multiwavelet system, this vectorization procedure can be simplified as an $r$-fold downsampling process, demonstrated as follows. Defining the analyzed signal as $S=\left[s_{1}, s_{2}, \ldots s_{N-1}\right]^{T}, S$ should be periodically extended until $N=\lceil N / r\rceil \times r$ if $N \bmod r \neq 0$. Then, the extended $S$ should be vectorized as an $r \times(N / r)$ matrix, i.e., $D_{0}^{(0)}=\left[D_{0}^{(0)}(0), D_{0}^{(0)}(1), \ldots, D_{0}^{(0)}(r-1)\right]^{T}$, where $D_{0}^{(0)}$ $(j)=\left[s_{0 \times r+j}, s_{1 \times r+j}, \ldots, s_{(N / r-1) \times r+j}\right]$ and $0 \leq j<r$. After establishing $D_{0}^{(0)}$ with the vectorization process, $D_{l}^{(k)}$ at each level can be calculated by repeatedly performing the decomposition process in Eq. (5). Figure 7 shows illustrative results of decomposition by the $\mathrm{M}$-band multiwavelet packet transform of a remote sensing image. As can be seen, numbers of transform domain coefficients in different cases of $\mathrm{M}$ are the same as all the transforms are orthogonal.

As shown in Fig. 5, the fast algorithm of the proposed high-order balanced M-band MWPT mainly consists of convolutions and subsampling processes and is very similar to the Mallat algorithm generally used in classical wavelet transforms.

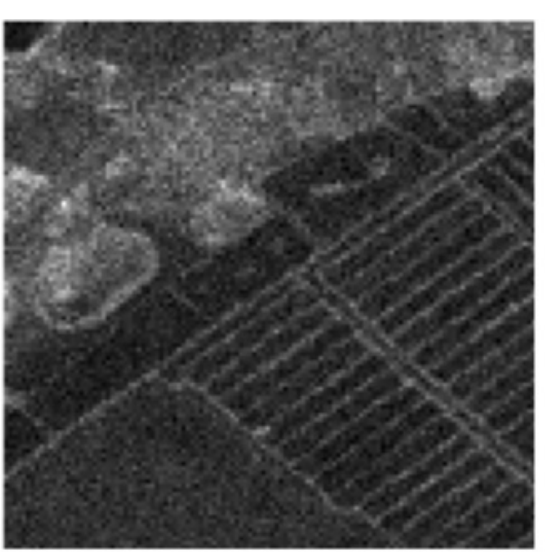

(a) Noisy image.

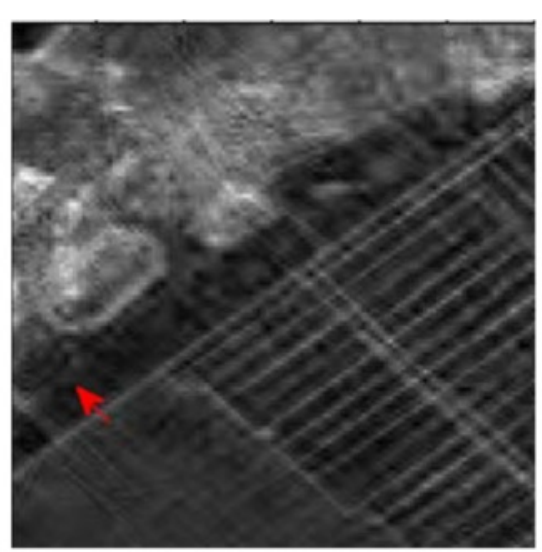

(b) Using the method of Liu et al. [16].

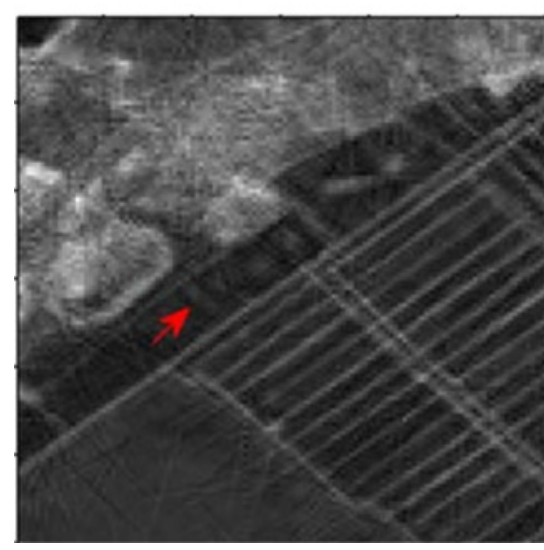

(c) Using the method of Ren et al. [25].

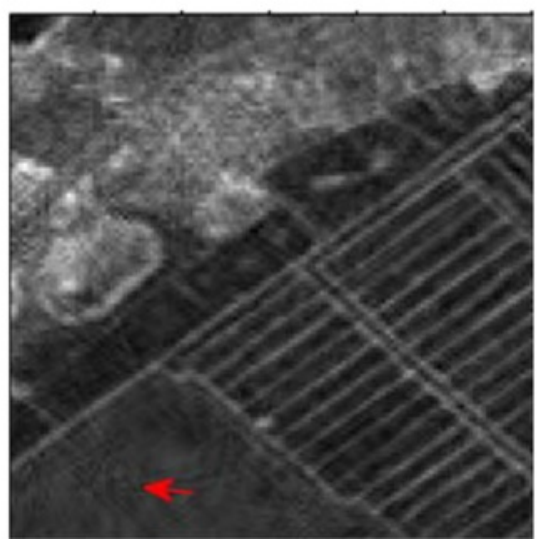

(d) Using the method of He et al. [23].

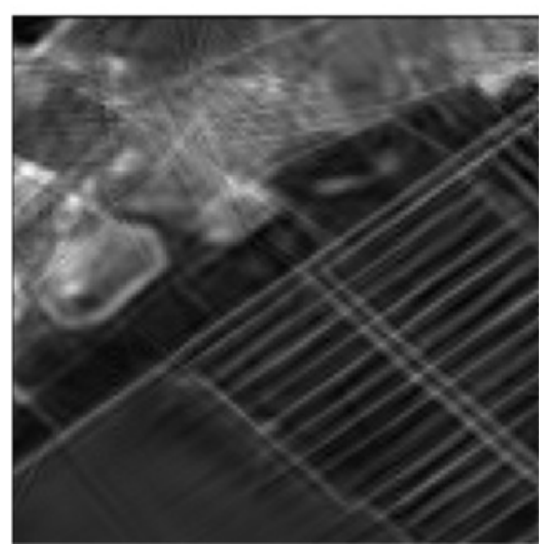

(e) Using our proposed method.

Fig. 9 Close-ups of the same part of the noisy image (a) and relevant denoising results obtained by different methods (b-e), when mixing the original image in Fig. 8a with pattern A noise. The part covered is that shown in Fig. 8, the same below 


\section{MWPT-based remote sensing image denoising 3.1 Denoising algorithm}

Many previously proposed wavelet transform-based denoising approaches can be introduced to MWPTbased remote sensing image denoising, notably the wavelet shrinkage method proposed by Donoho [2] and widely advocated in later studies [6, 10, 19, 22]. We applied this simple denoising scheme (rather than more complex shrinkage schemes) to compare the denoising performance of our transform and other traditional wavelet transforms. First, we applied the proposed MWPT for multiscale decomposition of noisy images then used soft thresholding to shrink the transform domain coefficients [2]. The threshold (universally) used was $T=\sigma \times \sqrt{2 \times \ln (N)}$, where $N$ is the number of pixels in the original image, and $\sigma$ is the variance of an additive noise, which may be unknown. If so, we used a robust median estimator $\hat{\sigma}$ of $\sigma$, computed from the multiwavelet coefficients of high-pass subband at scale $k=1$ as shown in Eq. (7).
Finally, the proposed MWPT was applied for reconstruction using the shrunk coefficients.

$$
\hat{\sigma}=\operatorname{median}\left[\left\{\left|D_{l}^{(1)}\right|\right\}\right] / 0.6745,1 \leq l<M
$$

\subsection{Denoising performance comparison}

Extensive simulations were carried out using both synthetic and real noisy remote sensing images to investigate the performance of the proposed high-order balanced MWPT-based denoising method. The high-order balanced M-band multiwavelet systems constructed in [26] were used here to construct our transform (i.e., MWPT with $\mathrm{M}>2$ and $\rho>0)$. We compared the denoising performance of our transform with those of the MWPT schemes proposed in recently relevant works, including the 2-band MWPT proposed by Liu et al. [16] (MWPT with $\mathrm{M}=2$ and $\rho=0)$, the balanced 2-band MWPT proposed by $\mathrm{He}$ et al. [23] (MWPT with $M=2$ and $\rho>0$ ), and the M-band MWT proposed by Ren et al. [25]. Using the algorithms in

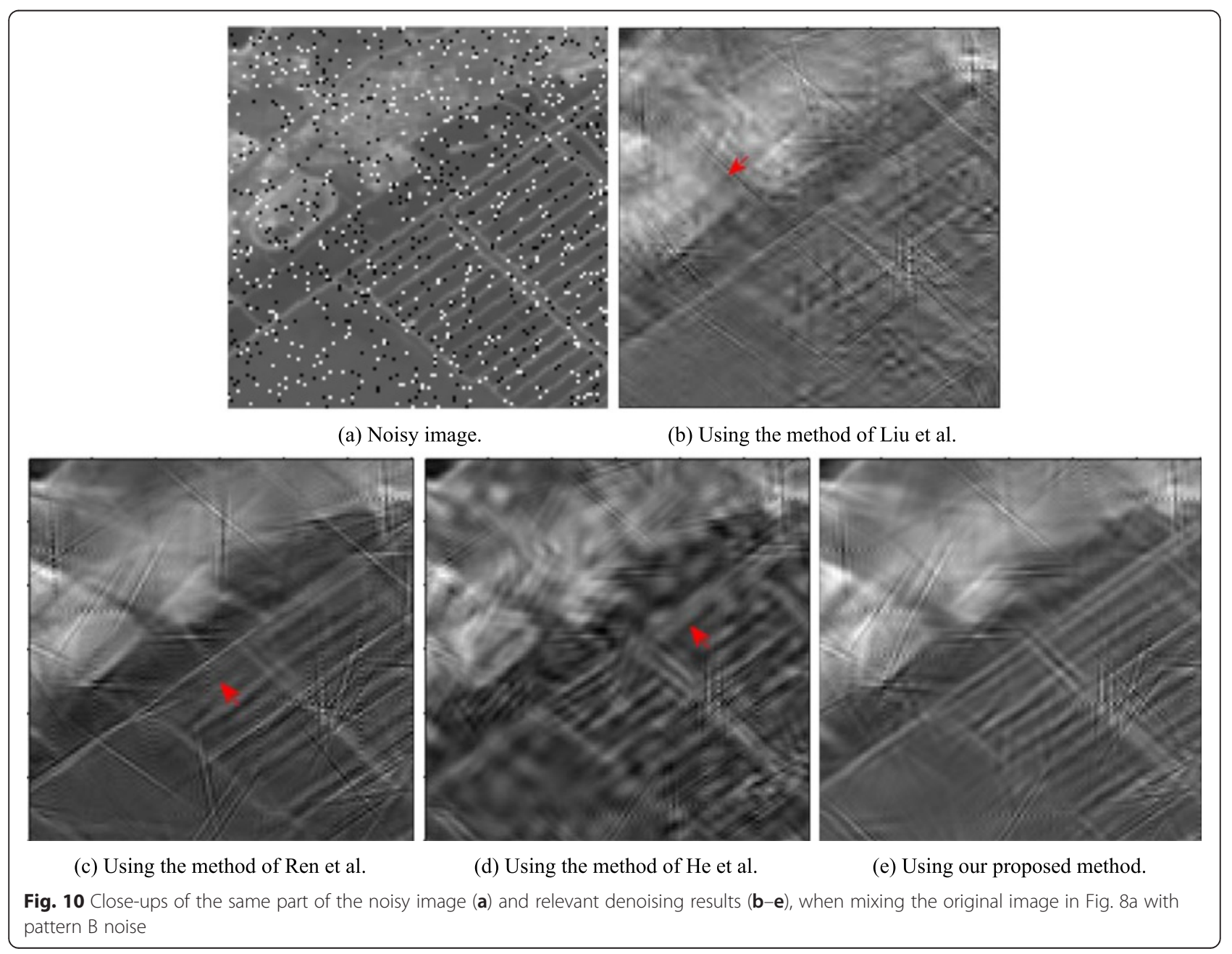


Section 2, the M-band MWT was developed into M-band MWPT (MWPT with $M>2$ and $\rho=0$ ) here for a fair comparison. The denoising strategies applied in these approaches are the same as those described in Section 3.1, apart from minor differences in the transforms per se.

\subsubsection{Synthetic noisy data-based analysis}

Various remote sensing images were used as original noiseless images for generating the synthetic noisy images. One of the test images is shown in Fig. 8a. This image, collected from the panchromatic band of the SPOT-5 satellite, covers an area in Yantai, China. As seen, it includes rich textures and edges. Figure $8 \mathrm{~b}$ displays a close-up of the image and shows the details of its textures.

To test the denoising performance to different patterns of noise attack, four kinds of noise are mixed with the original images as follows.

Pattern A: multiplicative noise. Multiplicative noise is added to the original noiseless image $I$ with the equation $J=I+V \times I$, where $V$ is uniformly distributed random noise with zero mean and variance 0.1.

Pattern B: salt and pepper noise. Salt and pepper noise is added to the original noiseless image $I$ with the noise density equals to 0.2 .

Pattern C: Gaussian white noise. Gaussian white noise is added to the original noiseless image $I$ with zero mean and variance 5 .

Pattern D: Poisson noise. Poisson noise is generated from the image itself instead of adding artificial noise to the original noiseless image $I$.

Results obtained with all the test images are similar. Thus, results obtained using the image in Fig. 8a are presented here to illustrate the denoising performance of the test methods. In the following figures, we display the denoising results of MWPT-based methods with typical cases of $(\mathrm{M}, \rho)$, including those with $(\mathrm{M}, \rho)$ equal to $(2,0),(4,0),(2,3)$, and $(4,2)$ (as proposed in $[16,23,25]$ and this work).

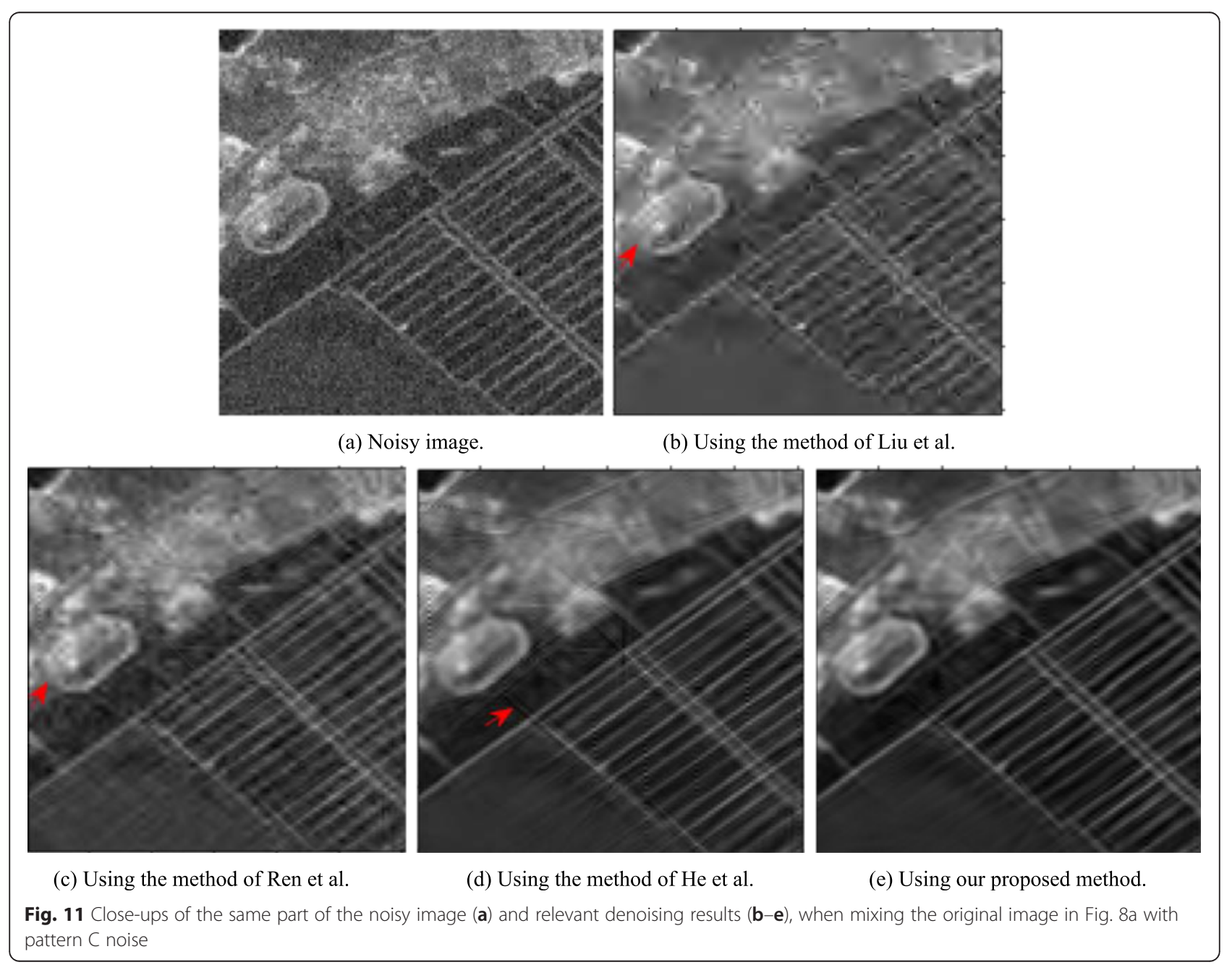




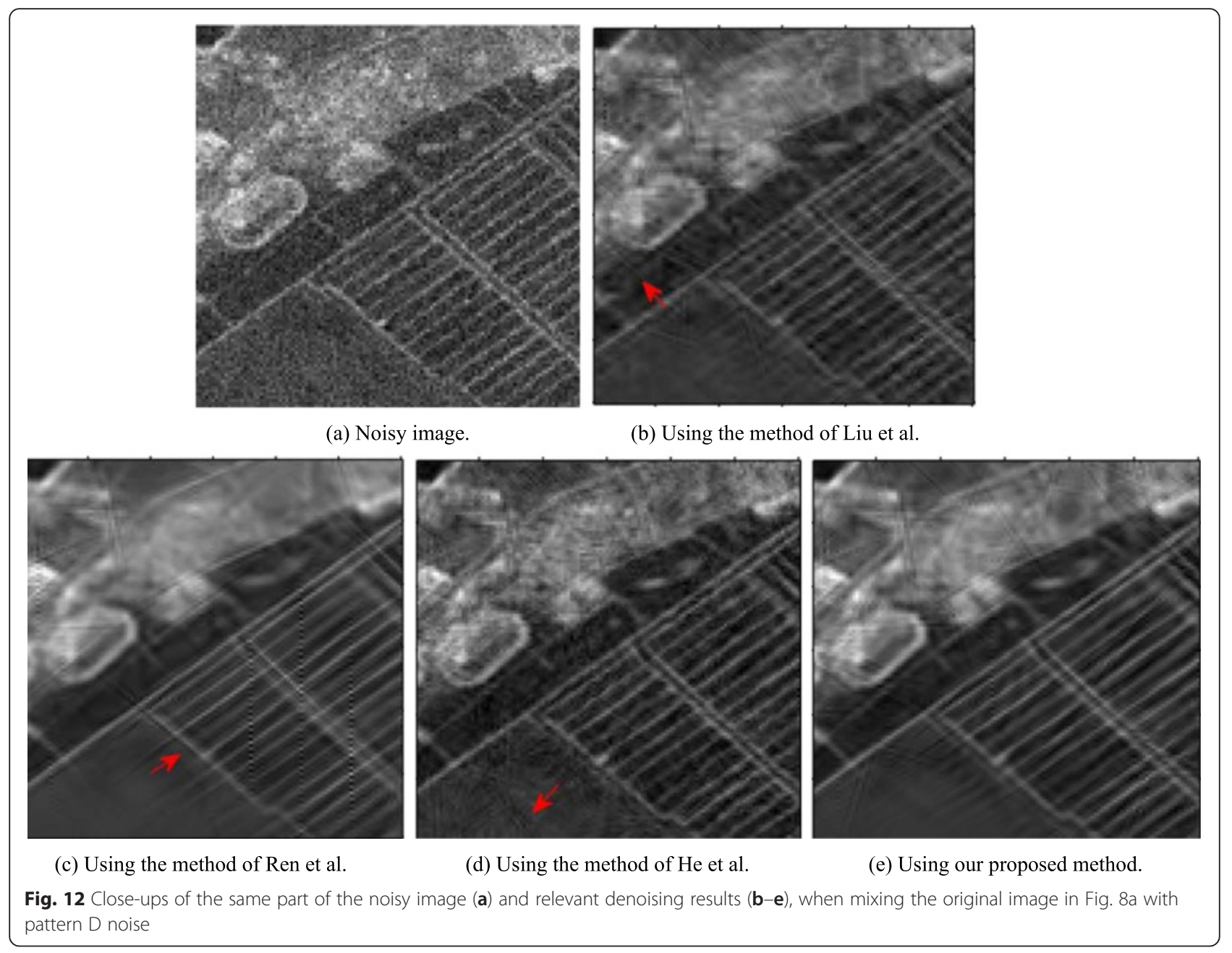

\section{A. Visual quality analysis}

As it is difficult to distinguish differences among the denoising images obtained using the tested methods at entire-image scale, we will consider the enlarged parts of the images obtained for the four noise patterns shown in
Figs. 9, 10, 11, and 12. In each case, the part covered is that shown in Fig. 8.

Figure 9 shows comparative close-ups when the original image is subjected to pattern A noise. As displayed in Fig. 9a, the image to be denoised is seriously contaminated by the multiplicative noise, compared with the

Table 1 Denoising indicator results obtained by applying all the test methods to denoise the above used image corrupted by different noise patterns

\begin{tabular}{lllllll}
\hline Noise patterns & Indicators & Noisy image & Method of Liu et al. & Method of Ren et al. & Method of He et al. & Method of our proposed method \\
\hline Pattern A & PSNR & 22.877 & 23.086 & 23.512 & 23.872 & 24.489 \\
& SSIM & 0.609 & 0.682 & 0.694 & 0.708 & 0.711 \\
Pattern B & PSNR & 15.651 & 18.466 & 19.043 & 19.630 & 21.632 \\
& SSIM & 0.427 & 0.523 & 0.552 & 0.547 & 0.602 \\
Pattern C & PSNR & 34.203 & 35.094 & 35.581 & 35.922 & 36.580 \\
& SSIM & 0.892 & 0.926 & 0.949 & 0.938 & 0.972 \\
Pattern D & PSNR & 28.476 & 29.754 & 30.741 & 30.293 & 31.602 \\
& SSIM & 0.751 & 0.802 & 0.817 & 0.836 & 0.852 \\
\hline
\end{tabular}


noiseless counterpart in Fig. 8b. Figure 9b, c shows that although the methods of Liu et al. and Ren et al. reduce the noise, both blur some of the edges in Fig. 9a (e.g., those in the area arrowed). The method of He et al. preserves edges relatively well but at the same time introduces "artifacts" (especially where arrowed), as shown in Fig. 9d. By contrast, the proposed method with high $M$ and $\rho$ (i.e., $(4,2))$ desirably suppresses the noise, while well preserving the edges and textures in the noisy image, as shown in Fig. 9e.

Figure 10 shows the results for images contaminated by pattern $B$ noise. It can be seen that all of the methods effectively suppress the salt and pepper noise shown in Fig. 10a. However, our proposed method more desirably retains some fine structures in the noisy image, in comparison with the other test methods.

The results for images contaminated by pattern C noise are shown in Fig. 11. The method of Liu et al. suppresses the Gaussian white noise in the smooth area well, but some edges and textures are somewhat oversmoothed (e.g., where arrowed). The method of Ren et al. also undesirably preserves some edges, as shown in Fig. 11c. Figure $11 \mathrm{~d}$ shows that the method of $\mathrm{He}$ et al. retains edge information well but adds some "artifacts" (e.g., in the arrowed area). In contrast, our method both reduces the noise and preserves the edges in the noisy image well (see Fig. 11e).

The proposed method also shows desirable performance in suppressing Poisson noise. As shown in Fig. 12, there are clear differences in results provided by the tested methods, although they all improve the quality of the noisy image displayed in Fig. 12a well. Figure $12 \mathrm{~b}$ shows that the method of Liu et al. blurs some edges in Fig. 12a. The method of Ren et al. desirably reduces the noise but somewhat oversmooths some edges and textures (e.g., those arrowed). The method of $\mathrm{He}$ et al. retains the fine structures well but introduces some "artifacts," while as shown in Fig. 12e, our method with high $\mathrm{M}$ and $\rho$ provides slightly better results.

\section{B. Quantitative analysis}

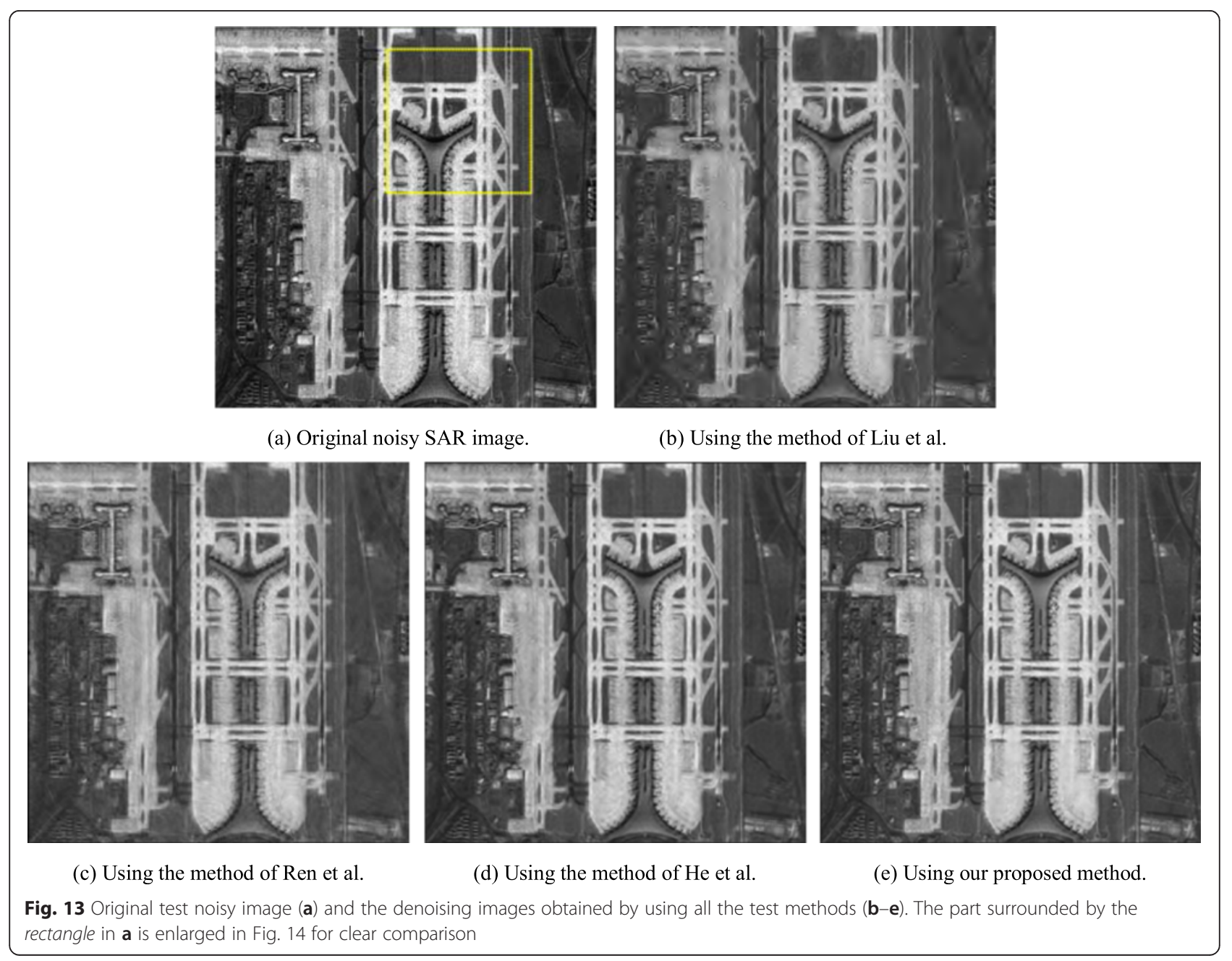




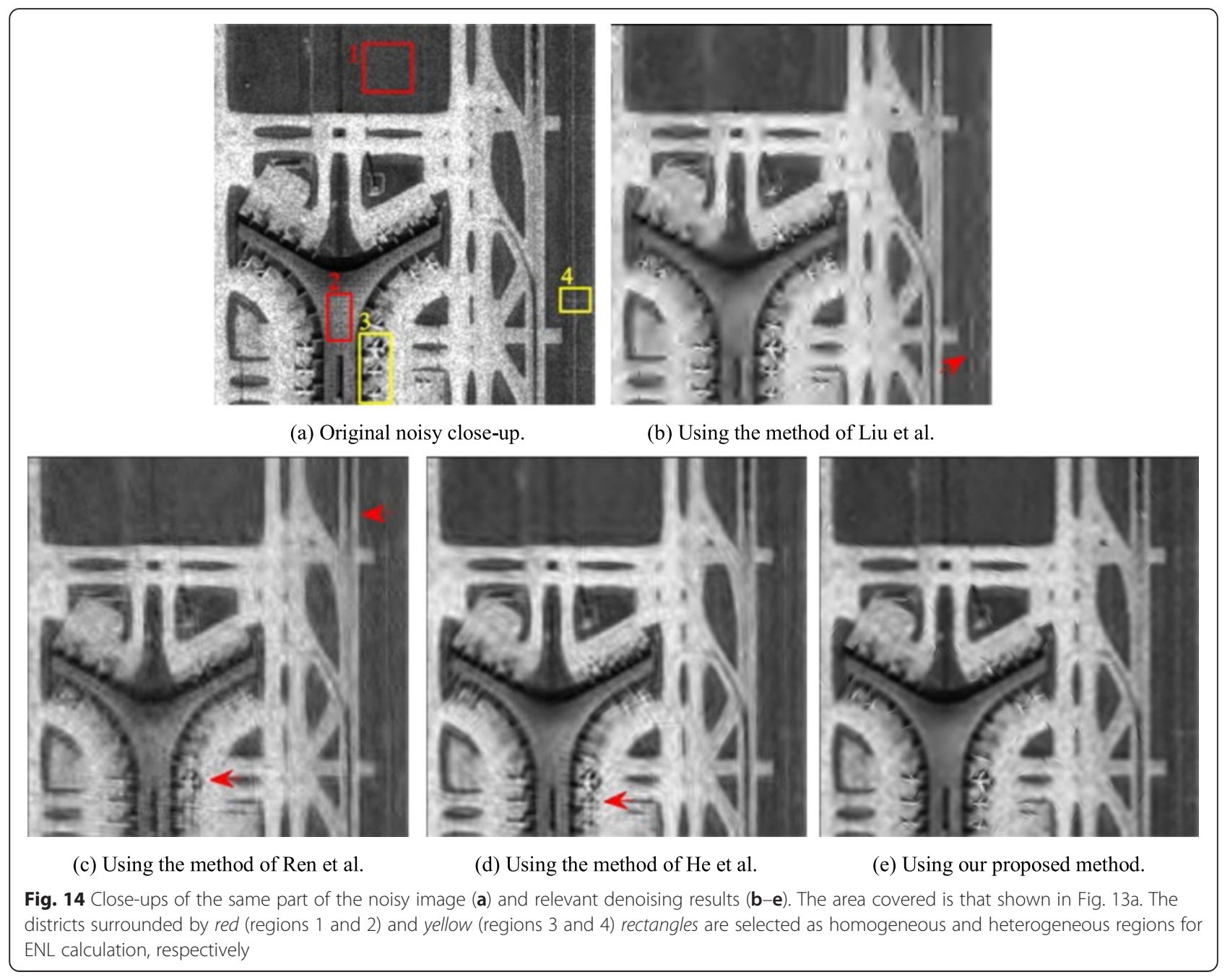

In order to analyze the tested methods' denoising effects quantitatively, we calculated peak signal-to-noise ratio (PSNR) and structural similarity (SSIM) values to assess from pixel- and structure-level fidelity aspects, respectively. SSIM is an image quality assessment index based on the human vision system and indicates degrees to which structural information in noisy image has been retained [27]. It is given by:

$$
\operatorname{SSIM}(x, y)=\frac{\left(2 \mu_{x} \mu_{y}+C_{1}\right)\left(2 \sigma_{x y}+C_{2}\right)}{\left(\mu_{x}^{2}+\mu_{y}^{2}+C_{1}\right)\left(\sigma_{x}^{2}+\sigma_{y}^{2}+C_{2}\right)}
$$

where $\mu_{x}$ and $\mu_{y}$ represent the average gray values of the noiseless reference image $x$ and the denoising image $y$, respectively, $\sigma_{x}$ and $\sigma_{y}$ represent the variances of $x$ and $y$, respectively, and $\sigma_{x y}$ represents the covariance between $x$

Table 2 ENL values obtained by all the test methods, using the data in Fig. 14

\begin{tabular}{llllllll}
\hline Region patterns & Regions & Size & \multicolumn{2}{l}{ ENL of original image and denoising results } & \\
\cline { 5 - 8 } & & & Original image & $\begin{array}{l}\text { Method of } \\
\text { He et al. }\end{array}$ & $\begin{array}{l}\text { Method of } \\
\text { He et al. }\end{array}$ & $\begin{array}{l}\text { Method of } \\
\text { He et al. }\end{array}$ & $\begin{array}{c}\text { Method of our } \\
\text { proposed method }\end{array}$ \\
\hline Homogeneous region & Region 1 (lawn) & $48 \times 48$ & 5.656 & 11.218 & 15.581 & 16.495 & 18.436 \\
& Region 2 (roadbed) & $24 \times 48$ & 4.941 & 9.481 & 8.490 & 10.572 & 12.683 \\
Heterogeneous region & Region 3 (plane) & $32 \times 36$ & 9.077 & 14.540 & 18.572 & 16.054 & 15.328 \\
& Region 4 (building) & $32 \times 24$ & 5.467 & 9.159 & 9.462 & 11.428 & 10.570 \\
\hline
\end{tabular}


Table 3 PSNR and SSIM values obtained by MWPT-based methods with different M and $\rho$ parameters, when using the test image in Fig. 8a and mixing Gaussian white noise with $\sigma=10$

\begin{tabular}{|c|c|c|c|c|c|c|c|c|}
\hline \multirow[t]{2}{*}{ Methods } & \multicolumn{4}{|l|}{ PSNR } & \multicolumn{4}{|l|}{ SSIM } \\
\hline & $\rho=0$ & $\rho=1$ & $\rho=2$ & $\rho=3$ & $\rho=0$ & $\rho=1$ & $\rho=2$ & $\rho=3$ \\
\hline MWPT with $M=2$ & 31.308 & 32.342 & 33.051 & 33.074 & 0.815 & 0.828 & 0.850 & 0.856 \\
\hline MWPT with $M=3$ & 32.159 & 33.065 & 33.552 & 33.537 & 0.820 & 0.846 & 0.871 & 0.860 \\
\hline MWPT with $M=4$ & 32.638 & 33.365 & 33.682 & 33.640 & 0.824 & 0.862 & 0.889 & 0.862 \\
\hline
\end{tabular}

and $y$. The symbols $C_{1}$ and $C_{2}$ are two constants which are used to prevent unstable results when either $\mu_{x}^{2}+\mu_{y}^{2}$ or $\sigma_{x}^{2}+\sigma_{y}^{2}$ very close to zero.

Table 1 presents PSNR and SSIM values obtained by applying all the test methods to denoise the above used image corrupted by different noise patterns. For every pattern, our proposed method consistently provides better results (higher PSNR and SSIM values) than the other test methods.

\subsubsection{Real noisy data-based analysis}

Synthetic aperture radar (SAR) images are inherently affected by multiplicative speckle noise, which generally affects the basic textures of SAR imagery [28]. Hence, we also chose real noisy SAR images without adding artificial noise to evaluate the performance of the proposed method. One of the test images was shown in Fig. 13a, covering an area of Beijing Airport, China, collected by airborne radars and provided by Chinese Academy of Science. As shown, it covers many ground objects and rich textures.

Figure 13b-e displays the denoising results obtained by applying all the test methods to the image shown in Fig. 13a. As can be seen, all the methods effectively reduce the speckle noise in the original image, especially in the smooth area (e.g., the surface of the runway). Figure 14 shows close-ups of the same part of the image obtained using each method for clear comparison. All of the MWPT-based methods clearly suppress speckle well, but those with low $\mathrm{M}$ or $\rho$ oversmooth images and thus blur many features (e.g., in the arrowed area). By contrast, the proposed 2-order balanced 4-band MWPTbased method preserves more structural details in the original image.
Since noise-free reference images were not available, equivalent number of looks (ENL) values of homogeneous regions were calculated. ENL is often applied to characterize the smoothing effect of denoising methods, given by:

$$
\operatorname{ENL}(x)=\mu_{x}^{2} / \sigma_{x}
$$

where the average $\mu_{x}$ and variance $\sigma_{x}$ are carried out over a target region $x[3,4,28]$. For a homogeneous region in a denoising image, the ENL value will simply reflect the degree to which the denoising method suppressed the noise in the region. However, for a heterogeneous region, the ENL value should not be too high, otherwise the method may oversmooth structural information regarding the region.

ENL values were calculated for two homogeneous regions (1 and 2 in Fig. 14a) and two heterogeneous regions (3 and 4) shown in Fig. 14b-e. The results, listed in Table 2, clearly show that the proposed method provides the highest ENL value for each homogeneous region and modest values for each heterogeneous region, demonstrating its superiority for both noise suppression and texture preservation. The results of the quantitative evaluation are also consistent with the visual assessment presented above.

\subsection{Influence of the $M, \rho$, and $k$}

The impacts of the band number $M$, the balanced order $\rho$, and the decomposition level $k$ on denoising performance when using the MWPT-based method can be summarized in more detail as follows.

Table 4 PSNR and SSIM values obtained by MWPT-based methods with different $M, \rho$, and $k$ parameters, when using the test image in Fig. 8a and mixing Gaussian white noise with $\sigma=10$

\begin{tabular}{|c|c|c|c|c|c|c|c|c|c|c|}
\hline \multirow[t]{2}{*}{ Methods } & \multicolumn{5}{|l|}{ PSNR } & \multicolumn{5}{|l|}{ SSIM } \\
\hline & $k=1$ & $k=2$ & $k=3$ & $k=4$ & $k=5$ & $k=1$ & $k=2$ & $k=3$ & $k=4$ & $k=5$ \\
\hline MWPT with $(M, \rho)=(2,3)$ & 29.862 & 31.658 & 32.981 & 33.072 & 32.685 & 0.751 & 0.795 & 0.830 & 0.856 & 0.848 \\
\hline MWPT with $(M, \rho)=(3,2)$ & 31.954 & 33.273 & 33.545 & 33.433 & 32.076 & 0.786 & 0.849 & 0.871 & 0.862 & 0.824 \\
\hline MWPT with $(M, \rho)=(4,2)$ & 32.729 & 33.480 & 33.683 & 33.248 & 31.716 & 0.801 & 0.866 & 0.889 & 0.854 & 0.790 \\
\hline
\end{tabular}


Table 5 Computational time (seconds) required by different MWPT-based methods, using the data in Fig. 8a

\begin{tabular}{lllll}
\hline $\begin{array}{l}\text { Size of } \\
\text { image }\end{array}$ & \multicolumn{4}{l}{ Computational times required by different methods } \\
\cline { 2 - 5 } & Method of Liu et al. & Method of Ren et al. & Method of He et al. & The proposed method \\
\hline $1000 \times 1000$ & 22.7 & 28.5 & 24.4 & 31.6 \\
\hline
\end{tabular}

(1)For every $M$ value between 2 and 4, as the balanced order $\rho$ rises within an appropriate range, the PSNR and SSIM provided by the MWPT-based method increase, as illustrated in Table 3 for the dataset considered above. These results clearly confirm the superiority of the high-order balanced M-band MWPT for preserving image features.

(2)As $M$ rises from 2 to 4 , the results of the MWPTbased method improve at each balanced order $\rho$. Table 3 shows the improvement when applied to the dataset in Fig. 8a. This further corroborates the advantages of M-band over 2-band multiwavelet systems.

(3) The MWPT-based method provides optimal results (at a given $\rho$ and $\mathrm{M}$ ) when the decomposition level $k$ is appropriate (3 or 4 in 3-band cases, and 2 or 3 in 4-band cases). This is illustrated by the PSNR and SSIM values in Table 4 for the images in Fig. 8a, showing that for several typical $\mathrm{M}$ and $\rho$ cases, 3-band and 4-band MWPTs provide optimal indicator values when $k=3$.

\subsection{Computational complexity analysis}

As the proposed transform is orthogonal, it adds little complexity and the computational costs of the proposed method are similar to those of methods based on traditional multiwavelets (e.g., GHM multiwavelet). Moreover, the appropriate decomposition levels for $\mathrm{M}>2$ cases are always less than that for the $M=2$ case reported in Section 3.2 , which further reduces the computational complexity of the proposed M-band MWPT-based method. These assertions were verified by the average computational times-in a MATLAB environment using a workstation with an Intel(R) Core $^{\mathrm{TM}}$ i5 CPU (3.2 GHz) and $4 \mathrm{~Gb}$ RAM-for the denoising cases reported in Section 3.2. Computation times of the denoising methods based on high-order balanced M-band MWPT are slightly longer than those of other MWPT-based methods but still reasonable (Table 5).

\section{Conclusions}

This work shows that using an appropriate higher balanced order or band number improves the denoising performance of multiwavelet packet transform-based methods at little additional computational cost. The high-order balanced M-band multiwavelet packet transform is also an effective scheme for other image processing tasks, such as texture analysis and edge extraction. This is because with an appropriate higher balanced order, the transform scheme provides more effective sparse image representation, a higher band number provides more flexible spatial-frequency domain partitioning, and the most suitable basis set for an analyzed signal can be selected for the multiwavelet packet transform scheme. We used a simple denoising strategy to evaluate the performance of the proposed transform scheme. To further improve its performance, other approaches that are more suitable for an M-band multiwavelet system should also be applied (e.g., taking into account the different frequency behaviors of the wavelets in a multiwavelet system in fusion rule selection).

Competing interests

The authors declare that they have no competing interests.

\section{Acknowledgements}

This work was supported by the Natural Science Foundation of Hebei Province, China (Grant No. D2015402159) and the National Natural Science Foundation of China (Grant Nos. 41330746 and 41171225), and also supported by the Natural Science Foundation of Hebei Province, China (Grant No. F2015402150), the Hebei Education Department, China (Grant Nos. ZD2014081 and ZD2015087), the Ministry of Agriculture's Special Funds for Scientific Study on Public Causes, China (Grant No. 201203062) and the National Natural Science Foundation of China (Grant Nos. 41371274 and 51309016). The author would like to thank I. W. Selesnick of Polytechnic Institute of New York University for many useful questions and comments.

\section{Author details}

${ }^{1}$ School of Information and Electrical Engineering, Hebei University of Engineering, Handan 056038, People's Republic of China. ${ }^{2}$ School of Electronic and Information Engineering, Bayin Guoleng Vocational and Technical College, Economic Development Zone, Korla 841000Xinjiang Uygur autonomous region, People's Republic of China. ${ }^{3}$ School of Resources and Environmental Engineering, Ludong University, Yantai 264025, People's Republic of China. ${ }^{4}$ College of Urban and Environmental Science, Northwest University, Xi'an 710127, People's Republic of China. Institute of Agricultural Water Conservancy, Changjiang River Scientific Research Institute, Wuhan 430012, People's Republic of China.

Received: 27 March 2015 Accepted: 17 December 2015

Published online: 27 January 2016

\section{References}

1. I Daubechies, Ten lectures on wavelets (SIAM, Philadelphia, 1992), pp. 313-319

2. DL Donoho, De-noising by soft-thresholding. IEEE T. Inform. Theory 41(3), 613-627 (1995)

3. H Xie, LE Pierce, FT Ulaby, SAR speckle reduction using wavelet denoising and Markov random field modeling. IEEE T. Geosci. Remote 40(10), 2196-2212 (2002)

4. A Achim, P Tsakalides, A Bezerianos, SAR image denoising via Bayesian wavelet shrinkage based on heavy-tailed modeling. IEEE T. Geosci. Remote 41(8), 1773-1784 (2003)

5. J Geronimo, D Hardin, PR Massopust, Fractal functions and wavelet expansions based on several scaling functions. J. Approx. Theory 78(3), 373-401 (1994)

6. V Strela, PN Heller, G Strang, P Topiwala, C Heil, The application of multiwavelet filterbanks to image processing. IEEE Trans. Image Process. 8(4), 548-563 (1999)

7. GY Chen, TD Bui, Multiwavelets denoising using neighboring coefficients. IEEE Signal Process. Let. 10(7), 211-214 (2003) 
8. TC Hsung, DP Lun, KC Ho, Optimizing the multiwavelet shrinkage denoising. IEEE T. Signal Process. 53(1), 240-251 (2005)

9. E Bala, A Ertuzun, A multivariate thresholding technique for image denoising using multiwavelets. EURASIP J. Applied Signal Process. 2005(1), 1205-1211 (2005)

10. AA Jumah, MG Ahamad, SA Ali, Denoising of medical images using multiwavelet transforms and various thresholding techniques. J. Signal Inf. Process. 4(1), 24-32 (2013)

11. JR Sveinsson, JA Benediktsson, Speckle reduction and enhancement of SAR images using multiwavelets and adaptive thresholding, in Proceedings of SPIE, Image and Signal Processing for Remote Sensing V (SPIE, Florence, 1999), p. 239

12. HH Wang, J Wang, J Zhang, Remote sensing image denoising by using discrete multiwavelet transform techniques, in Proceedings of SPIE, Remote Sensing and Infrared Devices and Systems (SPIE, Changchun, 2005), p. 603105

13. RR Coifman, MV Wickerhauser, Entropy-based algorithms for best basis selection. IEEE Trans. Inform. Theory 38(2), 713-718 (1992)

14. MB Martin, AE Bell, New image compression techniques using multiwavelets and multiwavelet packets. Image Process. IEEE T. 10(4), 500-510 (2001)

15. JW Wang, Multiwavelet packet transforms with application to texture segmentation. Electron. Lett. 38(18), 1021-1023 (2002)

16. ZG Liu, DB Zhang, DD Ma, De-noising and compression of power fault signals based on best multiwavelet packet, in Transmission and Distribution Conference and Exhibition: Asia and Pacific (IEEE/PES, Dalian, 2005), pp. 1-5

17. IW Selesnick, Balanced multiwavelets bases based on symmetric FIR filters. IEEE Trans. Signal Process. 48(1), 184-191 (2000)

18. J Lebrun, M Vetterli, High-order balanced multiwavelets: theory, factorization, and design. IEEE Trans. Signal Process. 49(9), 1918-1930 (2001)

19. $\mathrm{WZ} \mathrm{He}, \mathrm{AD} \mathrm{Wu}, \mathrm{GX}$ Song, Improvement of the resolution ratio of the seismic record by balanced biorthogonal multi-wavelet transform. Comput. Inf. Sci. 2005(3314), 379-384 (2005)

20. L Ghouti, A Bouridane, Image denoising using balanced multiwavelets and scale mixtures of Gaussians, in International Conference on Visual Information Engineering (IEE, Glasgow, 2005), pp. 101-105

21. LY Li, ZQ Wang, LX Song, Image denoising study based on balanced multiwavelet, in Proceeding of SPIE, Multispectral Image Acquisition and Processing (SPIE, Yichang, 2009), p. 749424

22. ZH Zeng, Construction of high performance balanced symmetric multifilter banks and application in image processing. J. Comput. 5(7), 1038-1045 (2010)

23. WZ He, AD Wu, Seismic data compression and denoising by balanced orthogonal multiwavelet packet, in Proceedings of the International Computer Congress on Wavelet Analysis and Its Applications, and Active Media Technology, vol. 1 (World Scientific, Chongqing, China, 2004), pp. 196-201

24. A Bhatti, $\mathrm{H}$ Ozkaramanli, M-band multiwavelets from spline super functions with approximation order, in International Conference on Acoustical Speech and Signal Processing, vol. 4 (IEEE, Orlando, 2002), pp. IV-4172

25. HZ Ren, YH Lan, Y Zhang, Research of multi-focus image fusion based on M-band multi-wavelet transform, in Fourth International Workshop on Advanced Computational Intelligence (IEEE, Wuhan, 2011), pp. 395-398

26. HJ Wang, QK Yang, R Li, High-order balanced M-band orthogonal multiwavelet: construction and application. Int. J. Phys. Sci. 7(12), 1884-1902 (2012)

27. Z Wang, AC Bovik, HR Sheikh, EP Simoncelli, Image quality assessment from error visibility to structural similarity. IEEE Trans. Image Process. 13(4), 600-612 (2004)

28. C Oliver, S Quegan, Understanding synthetic aperture radar images (Artech House, Boston, 1998), pp. 95-97

\section{Submit your manuscript to a SpringerOpen ${ }^{\circ}$ journal and benefit from:}

- Convenient online submission

- Rigorous peer review

Immediate publication on acceptance

- Open access: articles freely available online

- High visibility within the field

Retaining the copyright to your article

Submit your next manuscript at $\gg$ springeropen.com 